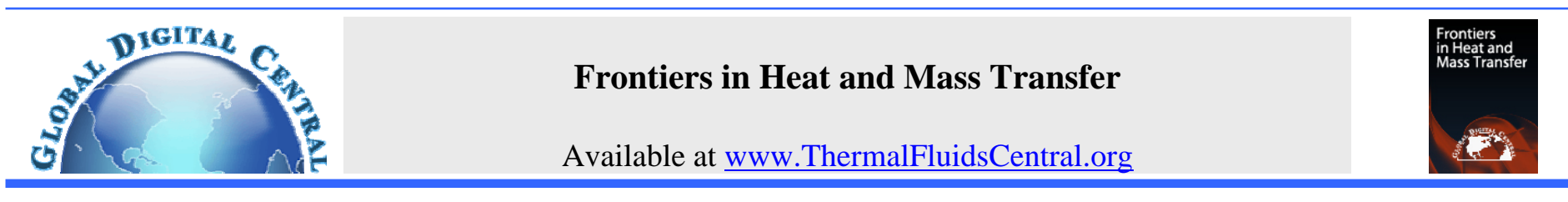

\title{
UNSTEADY HYDROMAGNETIC HEAT AND MASS TRANSFER NATURAL CONVECTION FLOW PAST AN EXPONENTIALLY ACCELERATED VERTICAL PLATE WITH HALL CURRENT AND ROTATION IN THE PRESENCE OF THERMAL AND MASS DIFFUSIONS
}

\author{
J. K. Singh ${ }^{\mathrm{a}, *}$, N. Joshi ${ }^{\mathrm{a}}$, S. G. Begum ${ }^{\mathrm{a}}$, C. T. Srinivasa ${ }^{\mathrm{b}}$ \\ ${ }^{a}$ Department of Mathematics, V. S. K. University, Ballari, Karnataka, 583105, INDIA \\ ${ }^{b}$ Department of Mathematics, V. G. F. G. C. Hiriyur, Karnataka, 577598, INDIA
}

\begin{abstract}
In the present analytical study, we have considered unsteady hydromagnetic heat and mass transfer natural convection flow of an electrically conducting, heat absorbing and chemically reacting fluid past an exponentially accelerated vertical plate in a uniform porous medium taking Hall current and rotation into account. The species concentration near the plate is considered to be varies linearly with time. Two particular cases for plate temperature are considered i.e. (i) plate temperature is uniform and (ii) plate temperature varies linearly with time and after some time it is maintained at uniform temperature. The coupled partial differential equations governing the fluid flow problem are solved analytically for fluid velocity, temperature and species concentration using Laplace transform method. To examine the physical characteristic of this problem the graphs for velocity, temperature, species concentration, skin friction, Nusselt number and Sherwood number distributions are computed and generated for various values of different pertinent flow parameters. It is observed that for small thermal diffusion, the free stream value of velocity and temperature are achieved nearer to the plate in comparison to that for large thermal diffusion.
\end{abstract}

Keywords: Hydromagnetic, natural convection, Hall current, rotation, permeability.

\section{INTRODUCTION}

Investigations made on hydromagnetic natural convection flow over a vertical surface has attracted attention of many researchers during past few decades due to its occurrence in many natural phenomenon and technological situations. Hydromagnetic natural convection flow of an electrically conducting, heat absorbing and chemically reacting fluid has been extensively studied due to its various practical applications viz. MHD pumps, MHD generators, oil exploration, nuclear power plants, gas turbines, aircrafts, space vehicles, geothermal energy extraction and fluids undergoing exothermic and endothermic chemical reaction etc. Raptis and Singh (1983) studied the effect of uniform transverse magnetic field on the free convection flow of an electrically conducting fluid past a uniformly as well as impulsively accelerated vertical plate. Hsieh et al. (1993) has obtained non similarity solution for mixed convection flow over a vertical flat plate embedded in a porous medium with variable surface temperature and variable surface heat flux. Kim (2000) studied unsteady MHD convective heat transfer flow past a impulsively moving semi-infinite vertical porous plate in the presence of a transverse magnetic field. An analytical solution for unsteady MHD convective heat and mass transfer flow past an infinite vertical porous plate embedded in porous medium with heat source/sink is presented by Kamel (2001). Israel-cookey and Tay (2002) studied unsteady flow of a radiating hydromagnetic fluid past an infinite flat plate when the radiative heat flux is presented in the general differential form. Israel-Cookey et al. (2003) investigated the influence of viscous dissipation and radiation on unsteady magnetohydrodynamic free convection flow past an infinite heated vertical plate in a porous medium with time dependent suction. An analytical study of unsteady MHD convective heat and mass transfer flow past a semi infinite vertical permeable moving plate in the presence of uniform transverse magnetic field and thermal and concentration buoyancy effects is carried by Chamkha (2004). A study on unsteady MHD flow of an electrically conducting and radiating fluid over a moving heated vertical porous plate with time dependent suction is presented by Israel-Cookey and Nwaigwe (2010). Seth and Ansari (2010) studied hydromagnetic natural convection flow of a viscous incompressible electrically conducting and heat absorbing fluid past an impulsively moving vertical plate with ramped wall temperature in a porous medium, in the presence of thermal diffusion. Pal and Talukdar (2011) studied the interaction of convection and thermal radiation on unsteady hydromagnetic heat and mass transfer flow past a semi infinite vertical moving plate embedded in a porous medium in the presence of heat absorption and first order chemical reaction. Magnetohydrodynamics free convection flow of a radiating and chemically reacting fluid past an impulsively moving plate with ramped wall temperature is discussed by Das (2012). Samiulhaq et al. (2012) presented an exact analysis of unsteady magnetohydrodynamic flow past an impulsively started vertical plate embedded in a porous medium in the presence of thermal diffusion and ramped wall temperature. Nandkeolyar et al. (2013) investigated unsteady hydromagnetic natural convection flow of a dusty fluid past an impulsively moving vertical plate with ramped temperature in the presence of thermal radiation. Kundu et al. (2014) considered unsteady MHD free convection mass transfer boundary layer flow of an incompressible electrically conducting fluid past an

\footnotetext{
" Corresponding author. Email: s.jitendrak@yahoo.com
} 
accelerated infinite vertical plate embedded in a porous medium with ramped wall temperature. Interaction of electromagnetic force to the Coriolis force plays a vital role in determining the flow features of hydromagnetic fluid flow problems. Study of influence of Coriolis force on hydromagnetic natural convection flow is significant because of several geophysical and fluid engineering problems are governed by interaction of Coriolis force and electromagnetic force. Keeping in view the importance of such study Bestman and Adjepong (1988) investigated unsteady hydromagnetic free convection flow near a moving infinite flat plate in a rotating medium with radiating heat transfer. Mbeledogu and Ogulu (2007) obtained analytical closed form solution of unsteady hydromagnetic natural convection flow of a rotating, incompressible, viscous Boussinesq fluid in the presence of radiative heat transfer and first order chemical reaction. The influence of radiation and rotation on unsteady hydromagnetic free convection flow past an impulsively moving infinite vertical plate with ramped temperature in a porous medium is investigated by Seth et al. (2013). Jonah Philliph et al. (2014) considered the problem of rotating magnetohydrodynamic heat and mass transfer free convection flow past an exponentially accelerated isothermal vertical plate in the presence of variable mass diffusion. It is observed that the effect of Hall current become significant when an ionized fluid with low density is permeated by a strong magnetic field (Sato, 1961). Seth et al. (2015) made an investigation on unsteady MHD natural convection flow past an exponentially accelerated vertical plate with ramped temperature through a porous medium with Hall effects and heat absorption. The combined effects of Hall current and rotation on unsteady MHD natural convection flow with heat and mass transfer past an impulsively moving vertical plate embedded in a porous medium in the presence of thermal and mass diffusion is presented by Seth et al. (2014).

In the problem studied by Jonah Philliph et al. (2014), the influence of Hall current, permeability of the medium, heat absorption/radiation and chemical reaction are not considered. Although these system parameters play significant role in determining the flow features of the hydromagnetic natural convection flows. Seth et al. (2014) extended the works of Seth et al. (2013) and Jonah Philliph et al. (2014) and investigated the influence of Hall current, permeability of the medium, heat absorption/radiation and chemical reaction on hydromagnetic natural convection flow past an impulsively moving isothermal vertical plate with uniform species concentration. Seth et al. (2015) considered the influence of Hall current, permeability and heat absorption on hydromagnetic natural convection flow past an exponentially accelerated vertical plate with ramped plate temperature but they have not considered the effect of rotation and species concentration in their problem. In the present research paper we have extended the work of Jonah Philliph et al. (2014) and Seth et al. (2015) and presented an analytical study on combined effects of Hall current and rotation on unsteady hydromagnetic heat and mass transfer natural convection flow past an exponentially accelerated vertical plate in a uniform porous medium with variable species concentration and uniform/variable plate temperature. It has been noticed from present analytical study that, Hall current accelerates fluid flow in the primary flow direction whereas Coriolis force decelerates it while both the Hall current and Coriolis force accelerate fluid flow in the secondary flow direction. Both the thermal diffusion and mass diffusion accelerate fluid flow in both the primary and secondary flow directions. Coriolis force enhances skin friction in both the primary and secondary flow directions while Hall current reduces skin friction in the primary flow direction and enhances in the secondary flow direction. For small thermal diffusion, the free stream value of velocity and temperature are achieved nearer to the plate in comparison to that for large thermal diffusion. When Darcian drag force is strong, the skin friction in the primary flow direction sharply decreasing whiles the skin friction in the secondary flow direction sharply increasing.

\section{MATHEMATICAL MODEL OF THE PROBLEM}

Cartesian coordinate system $\left(x^{\prime}, y^{\prime}, z^{\prime}\right)$ is considered as frame of reference for unsteady hydromagnetic heat and mass transfer flow of an incompressible, viscous, electrically conducting, heat absorbing and chemically reacting fluid past a vertical plate in a uniform porous medium. $x^{\prime}$-axis is chosen in such a way that it is along the plate in upward direction, $y^{\prime}$-axis is normal to the plane of the plate and $z^{\prime}$ axis is perpendicular to $x^{\prime} y^{\prime}$-plane. Flow past vertical plate is permeated by a uniform transverse magnetic field $B_{0}$ applied along $y^{\prime}$-direction. The fluid and the plate rotate unison as rigid body with uniform angular velocity $\vec{\Omega}$ about $y^{\prime}$-axis. Initially, at time $t^{\prime} \leq 0$, the fluid and plate are at rest. The temperature and species concentration at the plate are assumed to be $T_{\infty}^{\prime}$ and $C_{\infty}^{\prime}$ respectively. Suddenly, at time $t^{\prime}>0$, the plate is assumed to be exponentially accelerated with velocity $U_{0} e^{U_{0}^{2} t^{\prime} / v}$ in the vertical upward direction, where $U_{0}$ is a uniform velocity. At the same time the species concentration near the plate raised linearly with time $t^{\prime}$ to $C_{\infty}^{\prime}+\left(C_{w}^{\prime}-C_{\infty}^{\prime}\right) U_{0}^{2} t^{\prime} / v$. We have considered two particular cases for plate temperature:

(i) Uniform plate temperature: At time $t^{\prime}>0$, the plate temperature is raised to $T_{w}^{\prime}$.

(ii) Ramped plate temperature: At time $t^{\prime}>0$, the plate temperature is raised linearly with time $t^{\prime}$ to $T_{\infty}^{\prime}+\left(T_{w}^{\prime}-T_{\infty}^{\prime}\right) U_{0}^{2} t^{\prime} / v$ when $0<t^{\prime} \leq v / U_{0}^{2}$ and thereafter it is maintained at uniform temperature $T_{w}^{\prime}$ when $t^{\prime}>v / U_{0}^{2}$.

Since plate is electrically non-conducting and is of infinite extent along $x^{\prime}$ and $z^{\prime}$-directions, so all physical quantities will be function of $y^{\prime}$ and $t^{\prime}$ only. The physical model of the problem is presented in Fig.1.

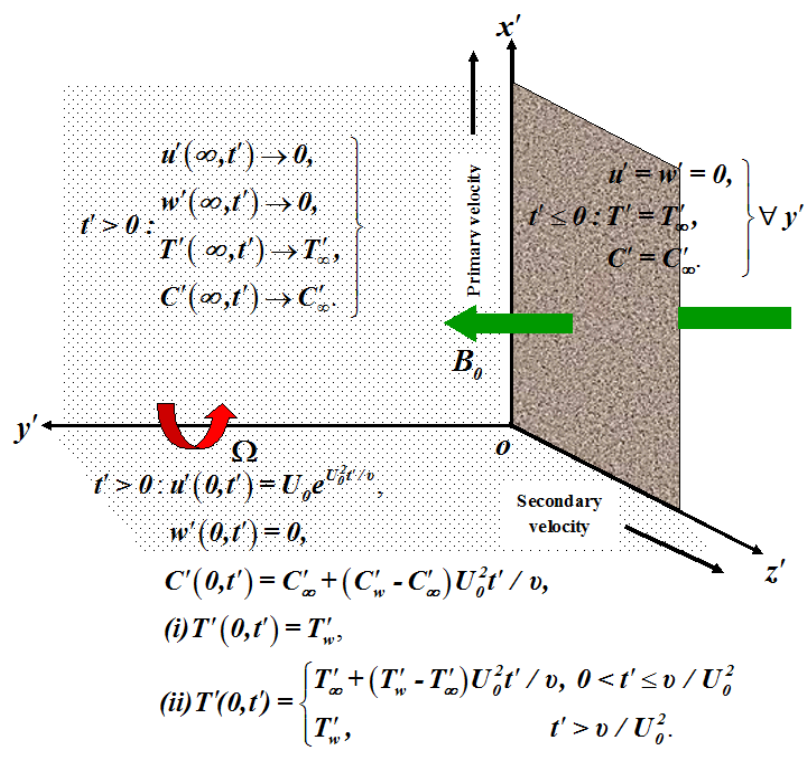

Fig. 1 Physical model of the problem.

The fluid considered is emitting/absorbing radiation but nonscattering. It is also assumed that a homogeneous chemical reaction of first order take place with constant rate $K^{\prime}$ between the diffusion species and fluid particles. There is no applied or polarization voltages imposed on the flow-field so the electric field $\vec{E}$ is assumed to be zero. The induced magnetic field generated by fluid motion is assumed to be negligible in comparison to applied one because fluid is assumed to be 
partially ionized and the magnetic Reynold's number $R_{m} \ll 1$ (Cramer and Pai, 1973). In the essence of above made assumptions the governing equations for unsteady hydromagnetic heat and mass transfer natural convection flow past an exponentially accelerated vertical plate in a porous medium in the presence of thermal and mass diffusions with Hall current and rotation under usual Boussinesq's approximation are given by

$$
\begin{aligned}
& \frac{\partial u^{\prime}}{\partial t^{\prime}}+2 \Omega w^{\prime}=v \frac{\partial^{2} u^{\prime}}{\partial y^{\prime 2}}-\frac{\sigma B_{0}^{2}}{\rho} \frac{\left(u^{\prime}+w^{\prime} m\right)}{\left(1+m^{2}\right)}+g \beta_{T}\left(T^{\prime}-T_{\infty}^{\prime}\right) \\
& +g \beta_{C}\left(C^{\prime}-C_{\infty}^{\prime}\right)-\frac{v u^{\prime}}{k^{\prime}}, \\
& \frac{\partial w^{\prime}}{\partial t^{\prime}}+2 \Omega u^{\prime}=v \frac{\partial^{2} w^{\prime}}{\partial y^{\prime 2}}-\frac{\sigma B_{0}^{2}}{\rho} \frac{\left(w^{\prime}-u^{\prime} m\right)}{\left(1+m^{2}\right)}-\frac{v w^{\prime}}{k^{\prime}}, \\
& \frac{\partial T^{\prime}}{\partial t^{\prime}}=\frac{k}{\rho C_{p}} \frac{\partial^{2} T^{\prime}}{\partial y^{\prime 2}}-\frac{Q_{0}}{\rho C_{p}}\left(T^{\prime}-T_{\infty}^{\prime}\right), \\
& \frac{\partial C^{\prime}}{\partial t^{\prime}}=D \frac{\partial^{2} C^{\prime}}{\partial y^{\prime 2}}-K^{\prime}\left(C^{\prime}-C_{\infty}^{\prime}\right),
\end{aligned}
$$

where $u^{\prime}$ and $w^{\prime}$ are velocity components in $x^{\prime}$ and $z^{\prime}$-directions respectively, $v$ is coefficient of viscosity, $\rho$ is fluid density, $m$ is Hall current parameter, $g$ is acceleration due to gravity, $\beta_{T}$ and $\beta_{C}$ are volumetric coefficient of thermal and species concentration expansions respectively, $T^{\prime}$ is fluid temperature, $C^{\prime}$ is species concentration, $k^{\prime}$ is permeability of flow medium, $k$ is thermal conductivity, $C_{p}$ is specific heat at constant pressure, $Q_{0}$ is heat absorption coefficient, $D$ is chemical molecular diffusivity and $K^{\prime}$ is chemical reaction parameter.

The initial and boundary conditions correspond to the two particular cases of fluid flows are given below:

For uniform plate temperature:

$$
\begin{aligned}
& t^{\prime} \leq 0: u^{\prime}=w^{\prime}=0, T^{\prime}=T_{\infty}^{\prime}, C^{\prime}=C_{\infty}^{\prime} \quad \forall y^{\prime}, \\
& t^{\prime}>0: \begin{cases}u^{\prime}=U_{0} e^{U_{0}^{2} t^{\prime} / v}, w^{\prime}=0, T^{\prime}=T_{w}^{\prime}, & \text { at } y^{\prime}=0 ; \\
C^{\prime}=C_{\infty}^{\prime}+\left(C_{w}^{\prime}-C_{\infty}^{\prime}\right) U_{0}^{2} t^{\prime} / v & \text { as } \mathrm{y}^{\prime} \rightarrow \infty . \\
u^{\prime} \rightarrow 0, w^{\prime} \rightarrow 0, T^{\prime} \rightarrow T_{\infty}^{\prime}, C^{\prime} \rightarrow C_{\infty}^{\prime} & \end{cases}
\end{aligned}
$$

For ramped plate temperature:

$$
\begin{aligned}
& t^{\prime} \leq 0: u^{\prime}=w^{\prime}=0, T^{\prime}=T_{\infty}^{\prime}, C^{\prime}=C_{\infty}^{\prime} \quad \forall y^{\prime}, \\
& t^{\prime}>0:\left\{\begin{array}{l}
u^{\prime}=U_{0} e^{U_{0}^{2} t^{\prime} / v}, w^{\prime}=0, C^{\prime}=C_{\infty}^{\prime}+\left(C_{w}^{\prime}-C_{\infty}^{\prime}\right) U_{0}^{2} t^{\prime} / v, \\
T^{\prime}=\left\{\begin{array}{l}
T_{\infty}^{\prime}+\left(T_{w}^{\prime}-T_{\infty}^{\prime}\right) U_{0}^{2} t^{\prime} / v \\
\text { when } 0<t^{\prime} \leq v / U_{0}^{2}, \\
T_{w}^{\prime} \quad \text { when } t^{\prime}>v / U_{0}^{2},
\end{array}\right. \\
u^{\prime} \rightarrow 0, w^{\prime} \rightarrow 0, T^{\prime} \rightarrow T_{\infty}^{\prime}, C^{\prime} \rightarrow C_{\infty}^{\prime}
\end{array} \text { as } y^{\prime} \rightarrow \infty .\right.
\end{aligned}
$$

We now define the following non-dimensional quantities:

$$
\begin{aligned}
& y=y^{\prime} U_{0} / v, u=u^{\prime} / U_{0}, w=w^{\prime} / U_{0}, t=t^{\prime} U_{0}^{2} / v, \\
& \theta=\left(T^{\prime}-T_{\infty}^{\prime}\right) /\left(T_{w}^{\prime}-T_{\infty}^{\prime}\right), C=\left(C^{\prime}-C_{\infty}^{\prime}\right) /\left(C_{w}^{\prime}-C_{\infty}^{\prime}\right) .
\end{aligned}
$$

Using above defined non-dimensional quantities and $F=u+i w$, the Eqs. (1)-(4) assume the following form

$$
\begin{aligned}
& \frac{\partial F}{\partial t}-2 i K^{2} F=\frac{\partial^{2} F}{\partial y^{2}}-\frac{M^{2} F}{1+m^{2}}(1-i m)+G_{T} \theta+G_{C} C-\frac{F}{k_{1}}, \\
& \frac{\partial \theta}{\partial t}=\frac{1}{P_{r}} \frac{\partial^{2} \theta}{\partial y^{2}}-\phi \theta, \\
& \frac{\partial C}{\partial t}=\frac{1}{S_{c}} \frac{\partial^{2} C}{\partial y^{2}}-K_{1} C,
\end{aligned}
$$

where $K^{2}=\Omega v / U_{0}^{2}$ is rotation parameter, $M^{2}=\sigma B_{0}^{2} v / \rho U_{0}^{2}$ is magnetic parameter, $G_{T}=v g \beta_{T}^{\prime}\left(T_{w}^{\prime}-T_{\infty}^{\prime}\right) / U_{0}^{3}$ is thermal Grashof number, $G_{C}=v g \beta_{C}^{\prime}\left(C_{w}^{\prime}-C_{\infty}^{\prime}\right) / U_{0}^{3}$ is solutal Grashof number, $k_{1}=k^{\prime} U_{0}^{2} / v^{2}$ is permeability parameter, $P_{r}=\rho C_{p} v / k$ Prandtl number, $\phi=Q_{0} v / \rho C_{p} U_{0}^{2}$ is heat absorption parameter, $S_{c}=v / D$ is Schmidt number and $K_{1}=K^{\prime} v / U_{0}^{2}$ is chemical reaction parameter.

The non-dimensional initial and boundary conditions for fluid flows are For uniform plate temperature:

$t \leq 0: F=0, \theta=0, C=0 \quad \forall y$,
$t>0: \begin{cases}F=e^{t}, \theta=1, C=t & \text { at } y=0 ; \\ F \rightarrow 0, \theta \rightarrow 0, C \rightarrow 0 & \text { as } y \rightarrow \infty .\end{cases}$

For ramped plate temperature:

$t \leq 0: F=0, \theta=0, C=0 \quad \forall y$,

$t>0: \begin{cases}F=e^{t}, C=t, \theta= \begin{cases}t & \text { when } 0<t \leq 1, \\ 1 & \text { when } t>1\end{cases} & \text { at } y=0 ; \\ F \rightarrow 0, \theta \rightarrow 0, C \rightarrow 0 & \text { as } y \rightarrow \infty .\end{cases}$

The mathematical model of the present physical problem is now complete and represented by Eqs. (9)-(11) subject to the initial and boundary conditions (12)-(13) and (14)-(15).

\section{SOLUTION OF THE PROBLEM}

Now we shall find the analytical solution of governing Eqs. (9)-(11) subject to the initial and boundary conditions (12)-(13) and (14)-(15) using Laplace transform method.

Laplace transform of Eqs. (9)-(11), using initial condition (12)/(14), yield

$$
\begin{aligned}
& \frac{d^{2} \bar{F}}{d y^{2}}-\left[s+\frac{1}{k_{1}}+\frac{M^{2}}{1+m^{2}}-i\left(2 K^{2}+\frac{m M^{2}}{1+m^{2}}\right)\right] \bar{F}+G_{T} \bar{\theta}+G_{C} \bar{C}=0, \\
& \frac{d^{2} \bar{\theta}}{d y^{2}}-P_{r}(s+\phi) \bar{\theta}=0, \\
& \frac{d^{2} \bar{C}}{d y^{2}}-S_{c}\left(s+K_{1}\right) \bar{C}=0,
\end{aligned}
$$


where

$\bar{F}(y, s)=\int_{0}^{\infty} e^{-s t} F(y, t) d t, \quad \bar{\theta}(y, s)=\int_{0}^{\infty} e^{-s t} \theta(y, t) d t$,

$\bar{C}(y, s)=\int_{0}^{\infty} e^{-s t} C(y, t) d t$, and $s(>0)$ is Laplace parameter.

The boundary conditions (13) and (15) after taking Laplace transform assume the following form

For uniform plate temperature:

$$
\left.\begin{array}{l}
\bar{F}=\frac{1}{s-1}, \bar{\theta}=\frac{1}{s}, \bar{C}=\frac{1}{s^{2}} \\
\bar{F} \rightarrow 0, \bar{\theta} \rightarrow 0, \bar{C} \rightarrow 0 \quad \text { at } y=0 ; \\
\text { as } \mathrm{y} \rightarrow \infty .
\end{array}\right\}
$$

For ramped plate temperature:

$$
\left.\begin{array}{ll}
\bar{F}=\frac{1}{s-1}, \bar{\theta}=\frac{1}{s^{2}}\left(1-e^{-s}\right), \bar{C}=\frac{1}{s^{2}} & \text { at } y=0 ; \\
\bar{F} \rightarrow 0, \bar{\theta} \rightarrow 0, \bar{C} \rightarrow 0 & \text { as } y \rightarrow \infty .
\end{array}\right\}
$$

Solving Eqs. (16)-(18) subject to the boundary conditions (19) and (20) and then inverting, the solution for fluid velocity, temperature and species concentration are obtained, and are represented by

For uniform plate temperature:

$$
\begin{aligned}
F(y, t)= & F_{1}\left(y, t, 1, m_{3}, 1\right)+\frac{G_{T}}{X_{1}\left(P_{r}-1\right)}\left\{F_{1}\left(y, t, 1, m_{3}, 0\right)\right. \\
& -F_{1}\left(y, t, P_{r}, \phi, 0\right)-F_{1}\left(y, t, 1, m_{3},-X_{1}\right) \\
& \left.-F_{1}\left(y, t, P_{r}, \phi,-X_{1}\right)\right\}-\frac{G_{C}}{X_{2}^{2}\left(S_{c}-1\right)}\left\{F_{1}\left(y, t, 1, m_{3}, 0\right)\right. \\
& -F_{1}\left(y, t, S_{c}, K_{1}, 0\right)-F_{1}\left(y, t, 1, m_{3},-X_{2}\right) \\
& +F_{1}\left(y, t, S_{c}, K_{1},-X_{2}\right)-X_{2}\left\{F_{2}\left(y, t, 1, m_{3}, 0\right)\right. \\
& \left.\left.-F_{2}\left(y, t, S_{c}, K_{1}, 0\right)\right\}\right\} \\
\theta(y, t)= & F_{1}\left(y, t, P_{r}, \phi, 0\right), \\
C(y, t)= & F_{2}\left(y, t, S_{c}, K_{1}, 0\right) .
\end{aligned}
$$

For ramped plate temperature:

$$
\begin{aligned}
F(y, t)= & F_{1}\left(y, t, 1, m_{3}, 1\right)+\frac{G_{T}}{\left(P_{r}-1\right)} \frac{1}{X_{1}^{2}}\left[\left\{X _ { 1 } \left(F_{2}\left(y, t, 1, m_{3}, 0\right)\right.\right.\right. \\
& \left.-F_{2}\left(y, t, P_{r}, \phi, 0\right)\right)-\left(F_{1}\left(y, t, 1, m_{3}, 0\right)-F_{1}\left(y, t, P_{r}, \phi, 0\right)\right. \\
& \left.\left.-F_{1}\left(y, t, 1, m_{3},-X_{1}\right)+F_{1}\left(y, t, P_{r}, \phi,-X_{1}\right)\right)\right\} \\
& -H(t-1)\left\{X_{1}\left(F_{2}\left(y, t-1,1, m_{3}, 0\right)-F_{2}\left(y, t-1, P_{r}, \phi, 0\right)\right)\right. \\
& -\left(F_{1}\left(y, t-1,1, m_{3}, 0\right)-F_{1}\left(y, t-1, P_{r}, \phi, 0\right)\right. \\
& \left.\left.\left.-F_{1}\left(y, t-1,1, m_{3},-X_{1}\right)+F_{1}\left(y, t-1, P_{r}, \phi,-X_{1}\right)\right)\right\}\right] \\
& +\frac{G_{C}}{\left(S_{c}-1\right)} \frac{1}{X_{2}^{2}}\left[X_{2}\left(F_{2}\left(y, t, 1, m_{3}, 0\right)-F_{2}\left(y, t, S_{c}, K_{1}, 0\right)\right)\right. \\
& -\left(F_{1}\left(y, t, 1, m_{3}, 0\right)-F_{1}\left(y, t, S_{c}, K_{1}, 0\right)\right. \\
& \left.\left.-F_{1}\left(y, t, 1, m_{3},-X_{2}\right)+F_{1}\left(y, t, S_{c}, K_{1},-X_{2}\right)\right)\right], \\
\theta(y, t)= & F_{2}\left(y, t, P_{r}, \phi, 0\right)-H(t-1) F_{2}\left(y, t-1, P_{r}, \phi, 0\right), \\
C(y, t)= & F_{2}\left(y, t, S_{c}, K_{1}, 0\right),
\end{aligned}
$$

where

$$
\begin{aligned}
& X_{1}=\frac{1}{P_{r}-1}\left(P_{r} \phi-m_{3}\right), X_{2}=\frac{1}{S_{c}-1}\left(S_{c} K_{1}-m_{3}\right), \\
& m_{3}=\frac{1}{k_{1}}+\frac{M^{2}}{1+m^{2}}-i\left(2 K^{2}+\frac{m M^{2}}{1+m^{2}}\right), \\
& \begin{aligned}
F_{1}(y, t, a, b, c)= & \frac{e^{c t}}{2}\left[e^{y \sqrt{a} \sqrt{b+c}} \operatorname{erfc}\left(\frac{y}{2} \sqrt{\frac{a}{t}}+\sqrt{(b+c) t}\right)\right. \\
& \left.+e^{-y \sqrt{a} \sqrt{b+c}} \operatorname{erfc}\left(\frac{y}{2} \sqrt{\frac{a}{t}}-\sqrt{(b+c) t}\right)\right], \\
F_{2}(y, t, a, b, c)= & \frac{e^{c t}}{2}\left[\left(t+\frac{y}{2} \sqrt{\frac{a}{b+c}}\right) e^{y \sqrt{a} \sqrt{b+c}} \operatorname{erfc}\left(\frac{y}{2} \sqrt{\frac{a}{t}}+\sqrt{(b+c) t}\right)\right. \\
+ & \left.\left(t-\frac{y}{2} \sqrt{\frac{a}{b+c}}\right) e^{-y \sqrt{a} \sqrt{b+c}} \operatorname{erfc}\left(\frac{y}{2} \sqrt{\frac{a}{t}}-\sqrt{(b+c) t}\right)\right] .
\end{aligned}
\end{aligned}
$$

Solutions (21) and (24) are not valid when $P_{r}=1$ and $S_{c}=1$ which corresponds to those fluids whose viscous, thermal and concentration boundary layer thickness are of same order of magnitude. For such fluids velocity can be obtained by putting $P_{r}=1$ and $S_{c}=1$ in Eqs. (17) and (18), solving Eqs. (16)-(18) subject to the boundary conditions (19) and (20) and then inverting. In this case of solutions for velocity field are given by

For uniform plate temperature:

$$
\begin{aligned}
F(y, t)= & F_{1}\left(y, t, 1, m_{3}, 1\right)+\frac{G_{T}}{\left(\phi-m_{3}\right)}\left\{F_{1}\left(y, t, 1, m_{3}, 0\right)-F_{1}(y, t, 1, \phi, 0)\right\} \\
& +\frac{G_{C}}{\left(K_{1}-m_{3}\right)}\left\{F_{2}\left(y, t, 1, m_{3}, 0\right)-F_{2}\left(y, t, 1, K_{1}, 0\right)\right\} .
\end{aligned}
$$

For ramped plate temperature:

$$
\begin{aligned}
F(y, t)= & F_{1}\left(y, t, 1, m_{3}, 1\right)+\frac{G_{T}}{\left(\phi-m_{3}\right)}\left\{F_{2}\left(y, t, 1, m_{3}, 0\right)-F_{2}(y, t, 1, \phi, 0)\right. \\
& \left.-H(t-1)\left\{F_{2}\left(y, t-1,1, m_{3}, 0\right)-F_{2}(y, t-1,1, \phi, 0)\right\}\right\} \\
& +\frac{G_{C}}{\left(K_{1}-m_{3}\right)}\left\{F_{2}\left(y, t, 1, m_{3}, 0\right)-F_{2}\left(y, t, 1, K_{1}, 0\right)\right\} .
\end{aligned}
$$

\section{SKIN FRICTION}

The skin friction $\tau_{x}$ and $\tau_{z}$ in primary and secondary flow directions in case of uniform plate temperature and ramped plate temperature when $P_{r} \neq 1$ and $S_{c} \neq 1$, are given by

For uniform plate temperature:

$$
\begin{aligned}
\tau_{x}+i \tau_{z}= & F_{3}\left(t, 1, m_{3}, 1\right)+\frac{G_{T}}{X_{1}\left(P_{r}-1\right)}\left\{F_{3}\left(t, 1, m_{3}, 0\right)-F_{3}\left(t, P_{r}, \phi, 0\right)\right. \\
& \left.-F_{3}\left(t, 1, m_{3},-X_{1}\right)+F_{3}\left(t, P_{r}, \phi,-X_{1}\right)\right\} \\
& -\frac{G_{C}}{X_{2}^{2}\left(S_{c}-1\right)}\left\{F_{3}\left(t, 1, m_{3}, 0\right)-F_{3}\left(t, S_{c}, K_{1}, 0\right)\right. \\
& -F_{3}\left(t, 1, m_{3},-X_{2}\right)+F_{3}\left(t, S_{c}, K_{1},-X_{2}\right) \\
& \left.-X_{2}\left\{F_{4}\left(t, 1, m_{3}, 0\right)-F_{4}\left(t, S_{c}, K_{1}, 0\right)\right\}\right\} .
\end{aligned}
$$


For ramped plate temperature:

$$
\begin{aligned}
\tau_{x}+i \tau_{z}= & F_{3}\left(t, 1, m_{3}, 1\right)+\frac{G_{T}}{\left(P_{r}-1\right)} \frac{1}{X_{1}^{2}}\left[\left\{X _ { 1 } \left(F_{4}\left(t, 1, m_{3}, 0\right)\right.\right.\right. \\
& \left.-F_{4}\left(t, P_{r}, \phi, 0\right)\right)-\left(F_{3}\left(t, 1, m_{3}, 0\right)-F_{3}\left(t, P_{r}, \phi, 0\right)\right. \\
& \left.\left.-F_{3}\left(t, 1, m_{3},-X_{1}\right)+F_{3}\left(t, P_{r}, \phi,-X_{1}\right)\right)\right\} \\
& -H(t-1)\left\{X_{1}\left(F_{4}\left(t-1,1, m_{3}, 0\right)-F_{4}\left(t-1, P_{r}, \phi, 0\right)\right)\right. \\
& -\left(F_{3}\left(t-1,1, m_{3}, 0\right)-F_{3}\left(t-1, P_{r}, \phi, 0\right)\right. \\
& \left.\left.\left.-F_{3}\left(t-1,1, m_{3},-X_{1}\right)+F_{3}\left(t-1, P_{r}, \phi,-X_{1}\right)\right)\right\}\right] \\
& +\frac{G_{C}}{\left(S_{c}-1\right)} \frac{1}{X_{2}^{2}}\left[X_{2}\left(F_{4}\left(t, 1, m_{3}, 0\right)-F_{4}\left(t, S_{c}, K_{1}, 0\right)\right)\right. \\
& -\left(F_{3}\left(t, 1, m_{3}, 0\right)-F_{3}\left(t, S_{c}, K_{1}, 0\right)-F_{3}\left(t, 1, m_{3},-X_{2}\right)\right. \\
& \left.\left.+F_{3}\left(t, S_{c}, K_{1},-X_{2}\right)\right)\right],
\end{aligned}
$$

where

$$
\begin{aligned}
F_{3}(t, a, b, c)= & e^{c t}\left[\sqrt{a(b+c)}\{\operatorname{erf} c \sqrt{(b+c) t}-1\}-\sqrt{\left.\frac{a}{\pi t} e^{-(b+c) t}\right],}\right. \\
F_{4}(t, a, b, c) & =e^{c t}\left[\left(t \sqrt{a(b+c)}+\frac{1}{2} \sqrt{\frac{a}{b+c}}\right)\{\operatorname{erf} c \sqrt{(b+c) t}-1\}\right. \\
& \left.-\sqrt{\frac{a t}{\pi}} e^{-(b+c) t}\right] .
\end{aligned}
$$

The skin friction $\tau_{x}$ and $\tau_{z}$ in primary and secondary flow directions in case of uniform plate temperature and ramped plate temperature when $P_{r}=1$ and $S_{c}=1$, are given by

For uniform plate temperature:

$$
\begin{aligned}
\tau_{x}+i \tau_{z}= & F_{3}\left(t, 1, m_{3}, 1\right)+\frac{G_{T}}{\left(\phi-m_{3}\right)}\left\{F_{3}\left(t, 1, m_{3}, 0\right)-F_{3}(t, 1, \phi, 0)\right\} \\
& +\frac{G_{C}}{\left(K_{1}-m_{3}\right)}\left\{F_{4}\left(t, 1, m_{3}, 0\right)-F_{4}\left(t, 1, K_{1}, 0\right)\right\} .
\end{aligned}
$$

For ramped plate temperature:

$$
\begin{aligned}
\tau_{x}+i \tau_{z}= & F_{3}\left(t, 1, m_{3}, 1\right)+\frac{G_{T}}{\left(\phi-m_{3}\right)}\left\{F_{4}\left(t, 1, m_{3}, 0\right)-F_{4}(t, 1, \phi, 0)\right. \\
& \left.-H(t-1)\left\{F_{4}\left(t-1,1, m_{3}, 0\right)-F_{4}(t-1,1, \phi, 0)\right\}\right\} \\
& +\frac{G_{C}}{\left(K_{1}-m_{3}\right)}\left\{F_{4}\left(t, 1, m_{3}, 0\right)-F_{4}\left(t, 1, K_{1}, 0\right)\right\} .
\end{aligned}
$$

\section{NUSSELT NUMBER}

The Nusselt number $N_{u}$ in case of uniform plate temperature and ramped plate temperature are given by

For uniform plate temperature:

$$
N_{u}=F_{3}\left(t, P_{r}, \phi, 0\right) .
$$

For ramped plate temperature:

$$
N_{u}=F_{4}\left(t, P_{r}, \phi, 0\right)-H(t-1) F_{4}\left(t-1, P_{r}, \phi, 0\right) .
$$

\section{SHERWOOD NUMBER}

The Sherwood number $S_{h}$ in case of both uniform plate temperature and ramped plate temperature is given by

$$
S_{h}=F_{4}\left(t, S_{c}, K_{1}, 0\right)
$$

\section{RESULTS AND ANALYSIS}

To examine the physical characteristic of this problem the graphs for velocity, temperature, species concentration, skin friction, Nusselt number and Sherwood number distributions are computed and generated for various values of different pertinent flow parameters. In the numerical computation the boundary condition $y \rightarrow \infty$ is approximated by $y_{\max }$ which is sufficiently large for velocity, temperature and species concentration to approach their free stream value. It can be observed from Figs. 2-15 that the fluid velocity in the primary flow direction, fluid temperature and species concentration attain their maximum value at the surface of the plate while the fluid velocity in the secondary flow direction attains its maximum value in the vicinity of the plate and thereafter these are decreasing on increasing boundary layer parameter $y$ to approach their free stream values. Figs. 2 depict that in case of both the uniform and ramped plate temperature, fluid velocity in both the primary and secondary flow directions increase on increasing Hall current parameter $m$. This shows that Hall current tends to enhance fluid velocity in both the primary and secondary flow directions. The usual nature of Hall current is to induce fluid flow in the secondary flow direction, our result comply it. This result equally agrees with the result of Seth et al. $(2014,2015)$.

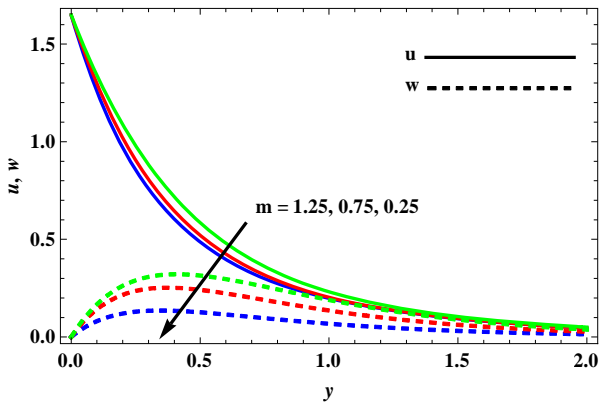

(a) Uniform plate temperature

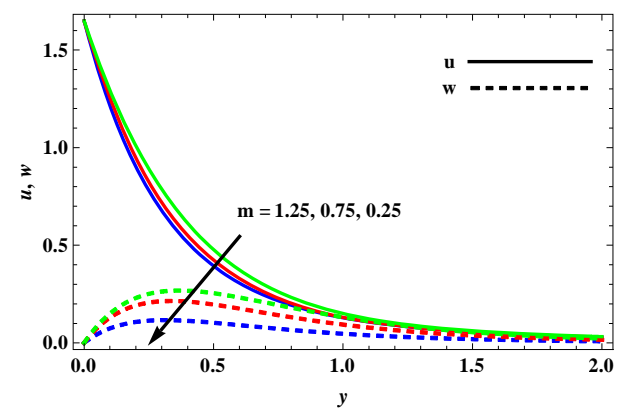

(b) Ramped plate temperat

Fig. 2 Velocity distribution when $K^{2}=1, M^{2}=9, k_{1}=0.3, G_{T}=4$, $G_{C}=5, P_{r}=0.71, S_{c}=0.22, \phi=1, K_{1}=0.2$ and $t=0.5$ : (a) uniform plate temperature and (b) ramped plate temperature. 
Figs. 3 display that in case of both the uniform and ramped plate temperature, fluid velocity in the primary flow direction decreases whereas the fluid velocity in the secondary flow direction increases on increasing rotation parameter $K^{2}$. This concludes that Coriolis force tends to reduce fluid velocity in the primary flow direction whereas it has reverse nature on the fluid velocity in the secondary flow direction. Similar to Hall current the usual nature of Coriolis force is to generate fluid flow in the secondary flow direction, our result agrees with it. This result also agrees with the result of Seth et al. (2013, 2014).

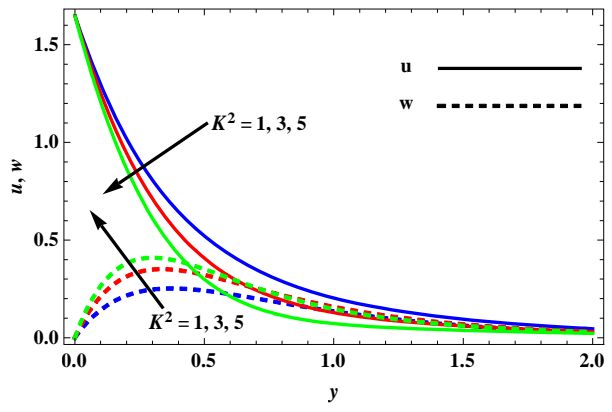

(a) Uniform plate temperature

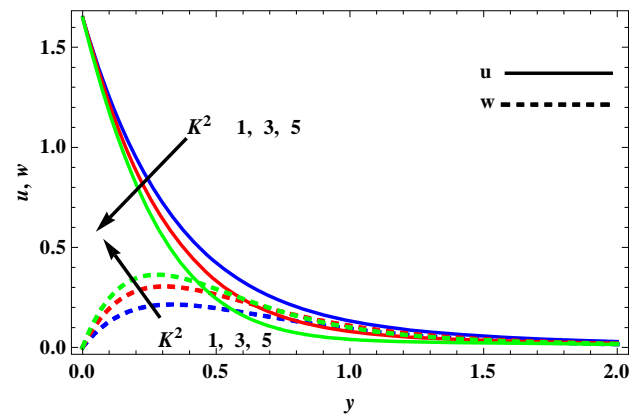

(b) Ramped plate temperature

Fig. 3 Velocity distribution when $m=0.75, M^{2}=9, k_{1}=0.3, G_{T}=4$, $G_{C}=5, P_{r}=0.71, S_{c}=0.22, \phi=1, K_{1}=0.2$ and $t=0.5:$ (a) uniform plate temperature and (b) ramped plate temperature.

Figs. 4 represent that in case of both the uniform and ramped plate temperature, the fluid in the primary flow direction decreases whereas the fluid velocity in the secondary flow direction increases in the vicinity of the plate and it decreases in the region away from the plate on increasing magnetic parameter $M^{2}$. This indicates that magnetic field (Lorentz force) tends to reduce fluid velocity in the primary flow direction and secondary flow direction in the region away from the plate whereas this nature is reversed on the fluid velocity in the secondary flow direction in the vicinity of the plate. Similar to drag force the usual nature of Lorentz force is to resist the main flow (primary flow), our result agrees with it. This result also corresponds to those of Kim (2000), Israle-Cookey and Nwaigwe (2010), Jonah Philliph et al. (2014) and Seth et al. (2014) which revealed that increased magnetic parameter decreases the primary fluid velocity. Figs. 5 demonstrate that in case of both the uniform and ramped plate temperature, fluid velocity in both the primary and secondary flow directions increase on increasing permeability parameter $k_{1}$, which implies that permeability tends to enhance fluid velocity in both the primary and secondary flow directions i.e. Darcian drag force tends to reduce fluid velocity in both the primary and secondary flow directions (when permeability is weak, Darcian drag force is strong).

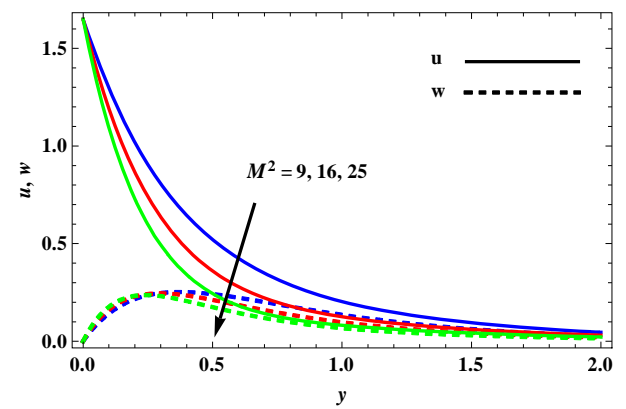

(a) Uniform plate temperature

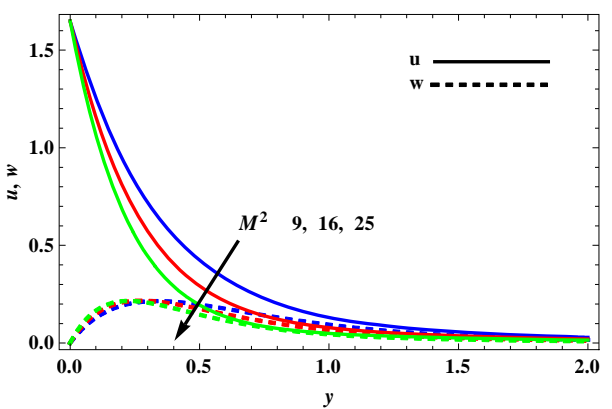

(b) Ramped plate temperature

Fig. 4 Velocity distribution when $m=0.75, K^{2}=1, k_{1}=0.3, G_{T}=4$, $G_{C}=5, P_{r}=0.71, S_{c}=0.22, \phi=1, K_{1}=0.2$ and $t=0.5$ : (a) uniform plate temperature and (b) ramped plate temperature.

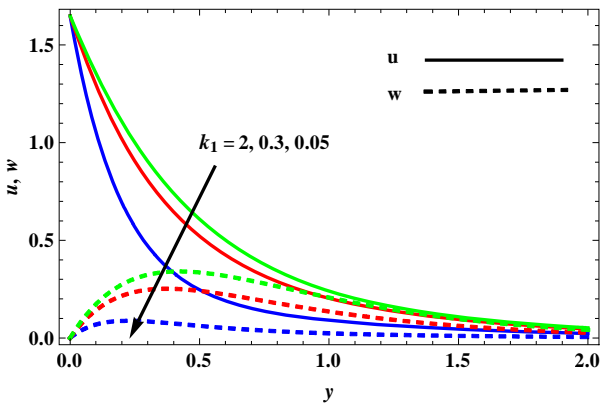

(a) Uniform plate temperature

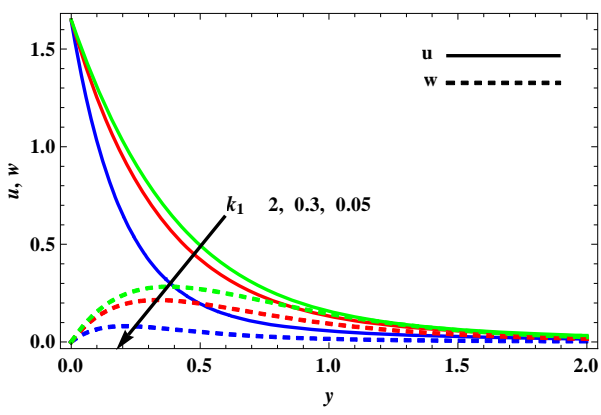

(b) Ramped plate temperature

Fig. 5 Velocity distribution when $m=0.75, K^{2}=1, M^{2}=9, G_{T}=4$, $G_{C}=5, P_{r}=0.71, S_{c}=0.22, \phi=1, K_{1}=0.2$ and $t=0.5$ : (a) uniform plate temperature and (b) ramped plate temperature. 


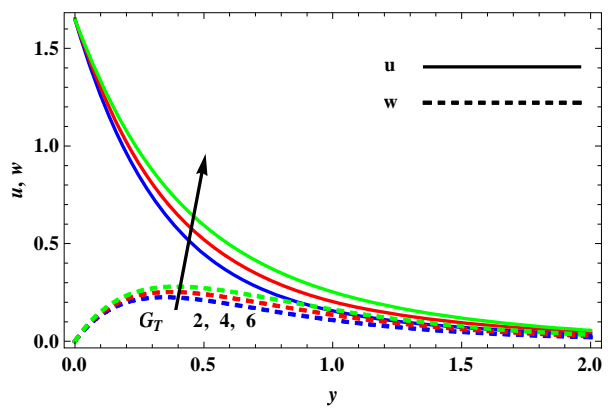

(a) Uniform plate temperature

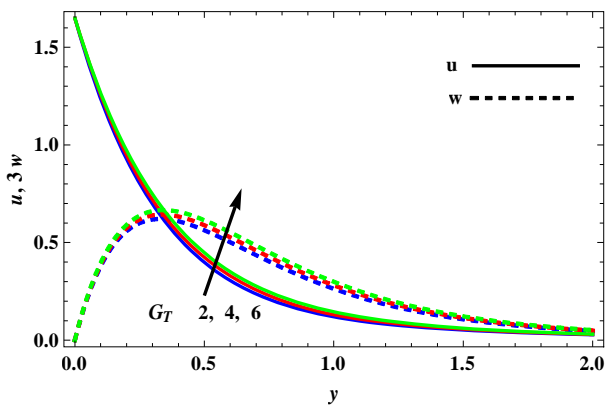

(b) Ramped plate temperature

Fig. 6 Velocity distribution when $m=0.75, K^{2}=1, M^{2}=9, k_{1}=0.3$, $G_{C}=5, P_{r}=0.71, S_{c}=0.22, \phi=1, K_{1}=0.2$ and $t=0.5:$ (a) uniform plate temperature and (b) ramped plate temperature.

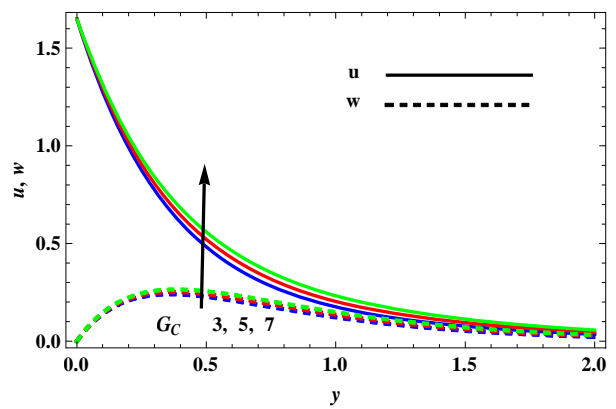

(a) Uniform plate temperature

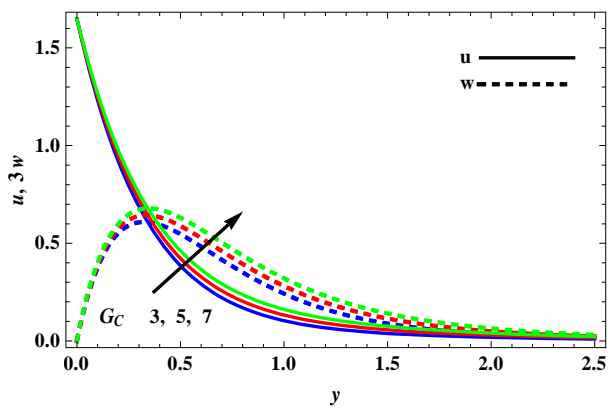

(b) Ramped plate temperature

Fig. 7 Velocity distribution when $m=0.75, K^{2}=1, M^{2}=9, k_{1}=0.3$, $G_{T}=4, P_{r}=0.71, S_{c}=0.22, \phi=1, K_{1}=0.2$ and $t=0.5$ : (a) uniform plate temperature and (b) ramped plate temperature.

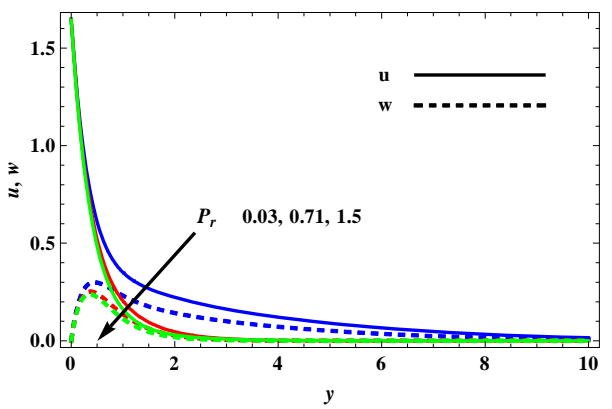

(a) Uniform plate temperature

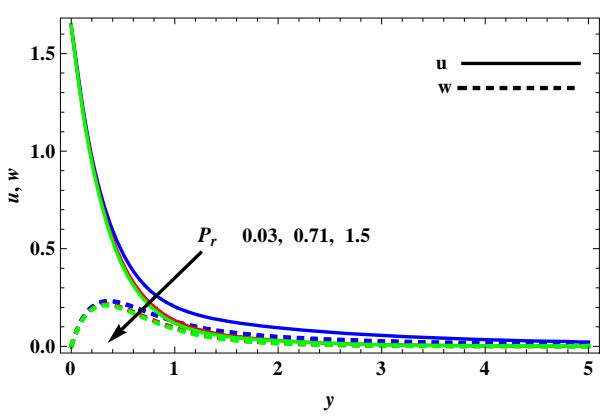

(b) Ramped plate temperature

Fig. 8 Velocity distribution when $m=0.75, K^{2}=1, M^{2}=9, k_{1}=0.3$, $G_{T}=4, G_{C}=5, S_{c}=0.22, \phi=1, K_{1}=0.2$ and $t=0.5$ : (a) uniform plate temperature and (b) ramped plate temperature.

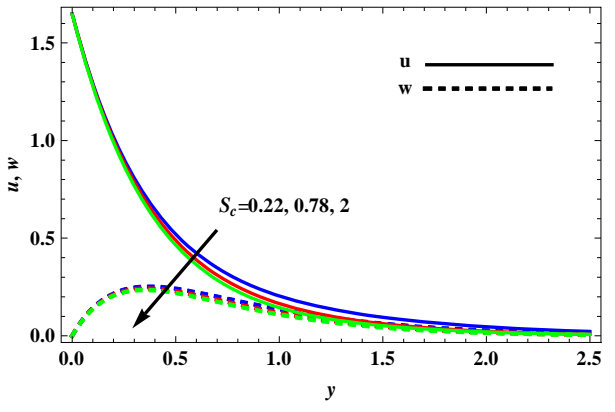

(a) Uniform plate temperature

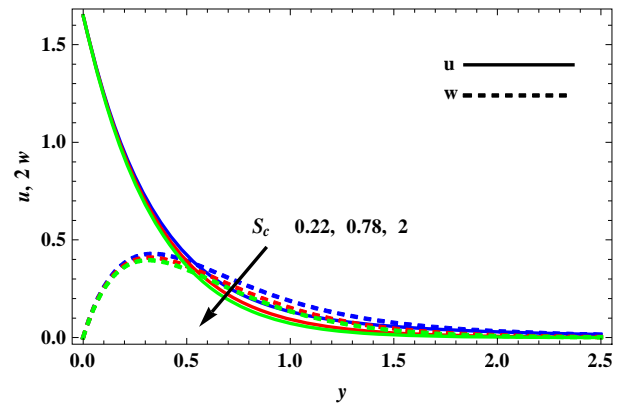

(b) Ramped plate temperature

Fig. 9 Velocity distribution when $m=0.75, K^{2}=1, M^{2}=9, k_{1}=0.3$, $G_{T}=4, G_{C}=5, P_{r}=0.71, \phi=1, K_{1}=0.2$ and $t=0.5$ : (a) uniform plate temperature and (b) ramped plate temperature. 


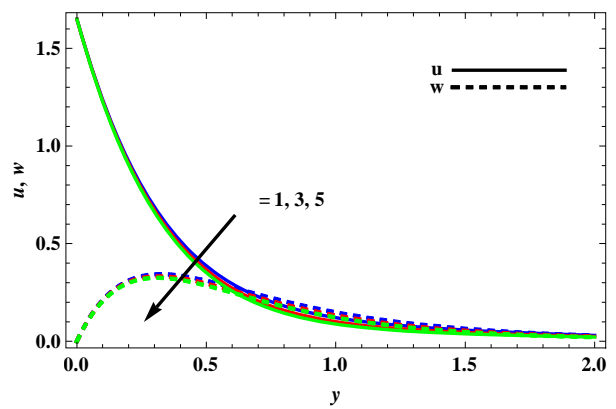

(a) Uniform plate temperature

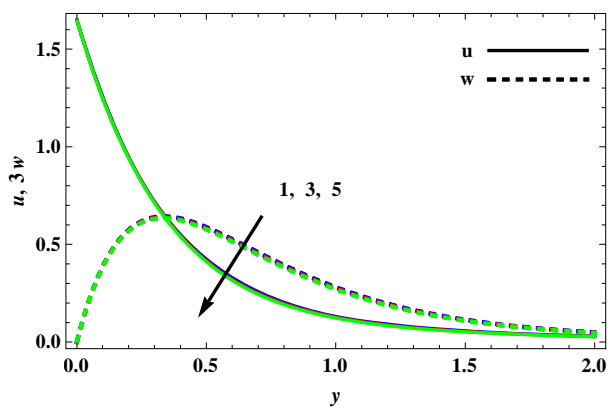

(b) Ramped plate temperature

Fig. 10 Velocity distribution when $m=0.75, K^{2}=1, M^{2}=9, k_{1}=0.3$, $G_{T}=4, G_{C}=5, P_{r}=0.71, S_{c}=0.22, K_{1}=0.2$ and $t=0.5$ : (a) uniform plate temperature and (b) ramped plate temperature.

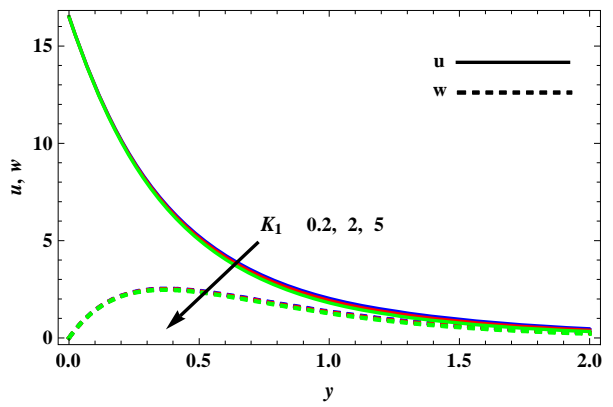

(a) Uniform plate temperature

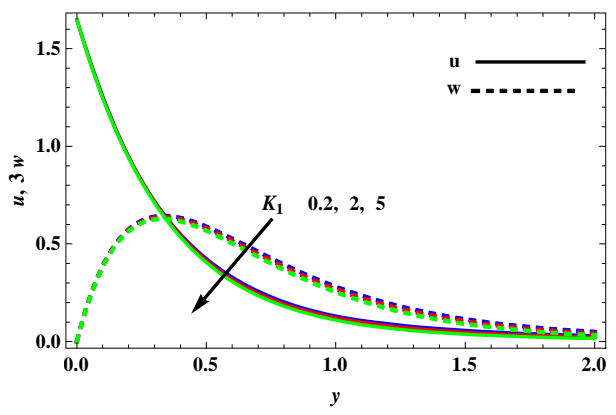

(b) Ramped plate temperature

Fig. 11 Velocity distribution when $m=0.75, K^{2}=1, M^{2}=9, k_{1}=0.3$, $G_{T}=4, G_{C}=5, P_{r}=0.71, S_{c}=0.22, \phi=1$ and $t=0.5$ : (a) uniform plate temperature and (b) ramped plate temperature.
Figs. 6 and 7 exhibit that incase of both the uniform and ramped plate temperature, fluid velocity in both the primary and secondary flow directions increase on increasing thermal Grashof number $G_{T}$ and solutal Grashof number $G_{C}$. This concludes that both the thermal and species concentration buoyancy forces tend to enhance fluid velocity in both the primary and secondary flow directions which agrees with the results obtained by Israle-Cookey and Nwaigwe (2010) and Seth et al. (2014). Figs. 8-11 show that in case of both the uniform and ramped plate temperature, fluid velocity in both the primary and secondary flow directions decrease on increasing Prandtl number $P_{r}$, Schemidt number $S_{C}$, heat absorption parameter $\phi$ and chemical reaction parameter $K_{1}$. Prandtl number and Schemidt number measures the relative strength of viscosity to the thermal diffusivity (thermal diffusion) and chemical molecular diffusivity (mass diffusion) respectively. Since there is inverse relation between Prandtl number and Schemidt number to the thermal diffusivity and chemical molecular diffusivity respectively. This implies that both the thermal and mass diffusions tend to enhance fluid velocity in both the primary and secondary flow directions whereas heat absorption and chemical reaction have reverse effect on these which agrees with the results of Jonah Philliph et al. (2014) and Seth et al. (2014, 2015).

Figs. 12 and 13 represent that in case of both the uniform and ramped plate temperature, fluid temperature decreases on increasing Prandtl number $P_{r}$ and heat absorption parameter $\phi$ which indicates that thermal diffusion tends to enhance fluid temperature whereas heat absoption has reverse effect on it. Figs .14 and 15 illustrate that in case of both the uniform and ramped plate temperature, species concentration decreases on increasing Schemidt number $S_{c}$ and chemical reaction parameter $K_{1}$ which concludes that mass diffusion tends to enhance species concentration whereas chemical reaction has reverse effect on it. This result comply the results of Jonah Philliph et al. (2014) and Seth et al. (2014). It is also observed from Figs. 8 and 12 that for small thermal diffusion, the free stream value of velocity and temperature are achieved nearer to the plate in comparison to the large thermal diffusion.

Figs. 16 depict that in case of both the uniform and ramped plate temperature, skin friction in both the primary and secondary flow directions increase on increasing rotation parameter $K^{2}$. Skin friction in the primary flow direction decreases whereas the skin friction in the secondary flow direction increases on increasing Hall current parameter $m$. This implies that Coriolis force tends to enhance skin friction in both the primary and secondary flow directions while Hall current tends to reduce skin friction in the primary flow direction whereas this effect is reversed on the skin friction in the secondary flow direction.Figs. 17 exhibit that in case of both the uniform and ramped plate temperature, skin friction in both the primary and secondary flow directions increase on increasing magnetic parameter $M^{2}$. Skin friction in the primary flow direction decreases whereas the skin friction in the secondary flow direction increases on increasing permeability parameter $k_{1}$. This reveals that magnetic field (Lorentz force) tends to enhance skin friction in both the primary and secondary flow directions while permeability tends to reduce skin friction in the primary flow direction whereas this effect is reversed on the skin friction in the secondary flow direction. It is also observed from Figs. 17 that Darcian drag force is strong, the skin friction in the primary flow direction decreases sharply whereas the skin friction in the secondary flow direction increases sharply and when Darcian drag force is weak, the skin friction in both the primary and secondary flow directions vary linearly with permeability. Figs. 18 show that in case of both the uniform and ramped plate temperature, skin friction in the primary flow direction decreases whereas skin friction in the secondary flow direction increases on increasing both the thermal Grashof number $G_{T}$ and solutal Grashof number $G_{C}$. This implies that both the thermal and 
species concentration buoyancy forces tend to reduce skin friction in the primary flow direction whereas these have reverse effect on the skin friction in the secondary flow direction.

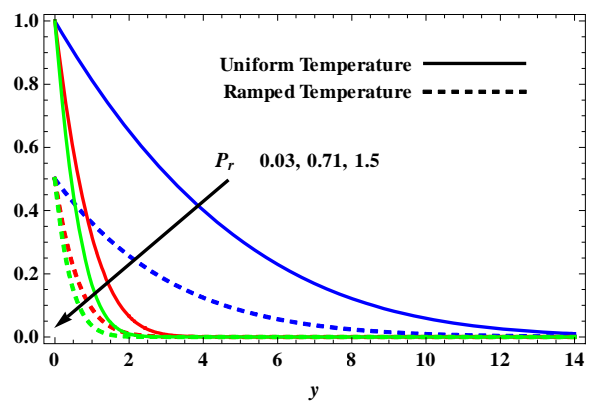

Fig. 12 Temperature distribution in case of both uniform and ramped plate temperature when $\phi=1$.

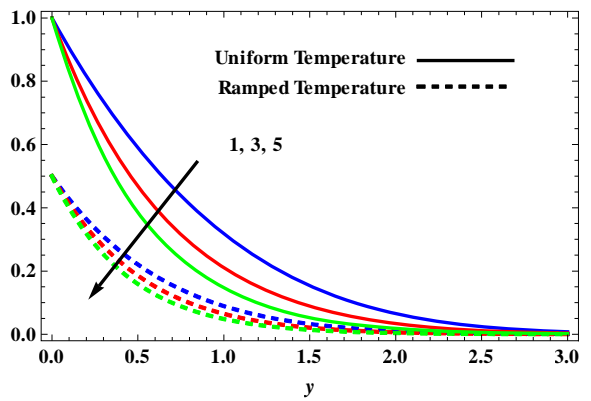

Fig. 13 Temperature distribution in case of both uniform and ramped plate temperature when $P_{r}=0.71$.

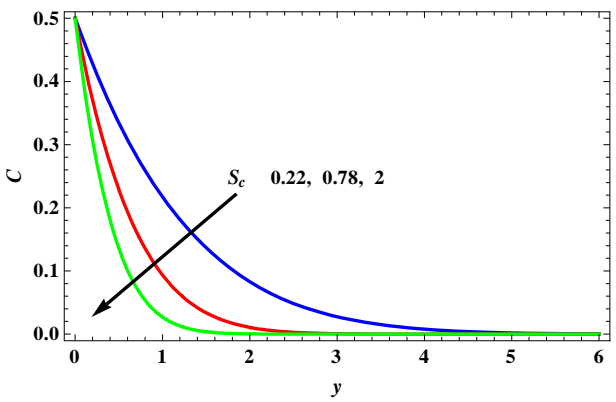

Fig. 14 Concentration distribution in case of both uniform and ramped plate temperature when $K_{1}=0.2$.

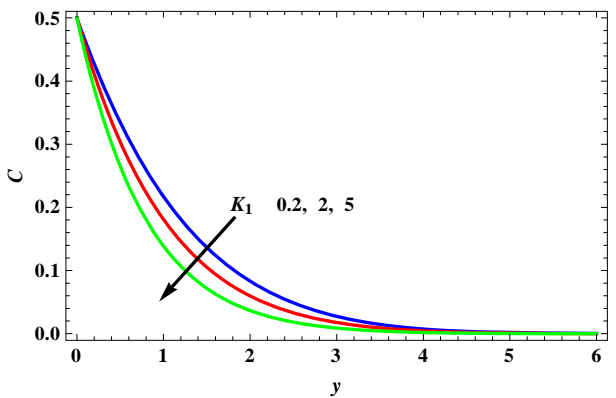

Fig. 15 Concentration distribution in case of both uniform and ramped plate temperature when $S_{c}=0.22$.

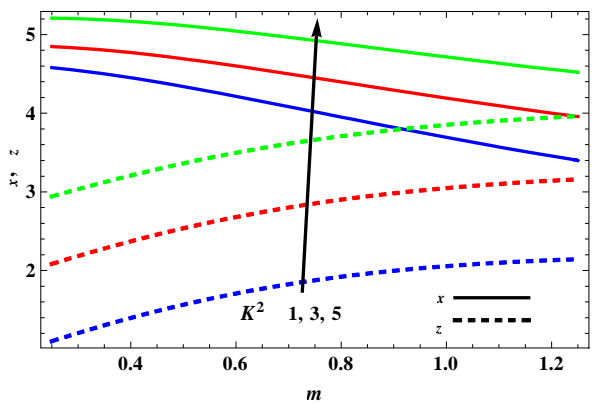

(a) Uniform plate temperature

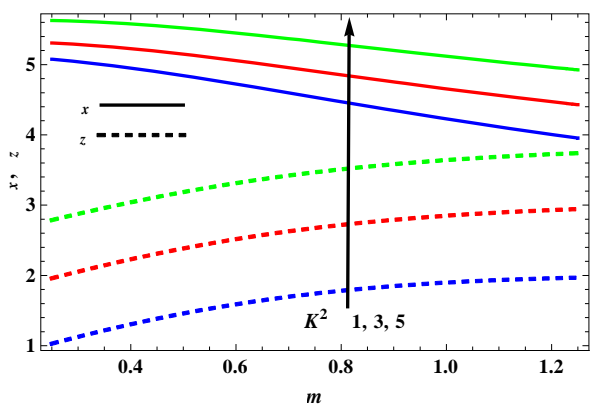

(b) Ramped plate temperature

Fig. 16 Skin friction distribution when $M^{2}=9, k_{1}=0.3, G_{T}=4$, $G_{C}=5, P_{r}=0.71, S_{c}=0.22, \phi=1, K_{1}=0.2$ and $t=0.5$ : (a) uniform plate temperature and (b) ramped plate temperature.

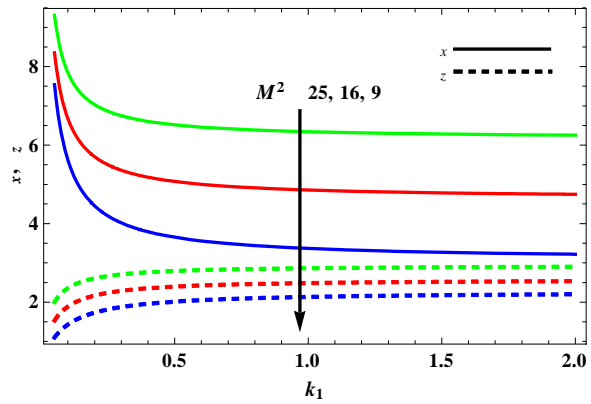

(a) Uniform plate temperature

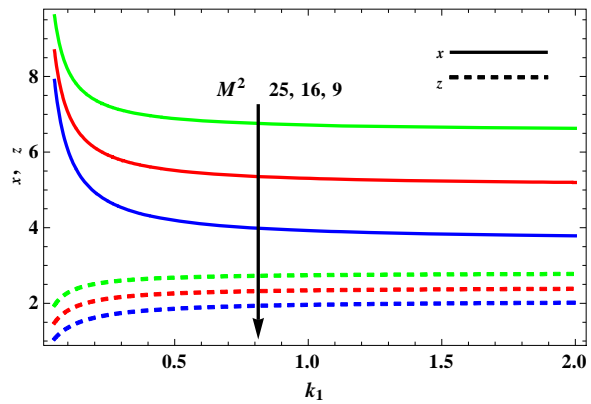

(b) Ramped plate temperature

Fig. 17 Skin friction distribution when $m=0.75, K^{2}=1, G_{T}=4$, $G_{C}=5, P_{r}=0.71, S_{c}=0.22, \phi=1, K_{1}=0.2$ and $t=0.5:$ (a) uniform plate temperature and (b) ramped plate temperature. 


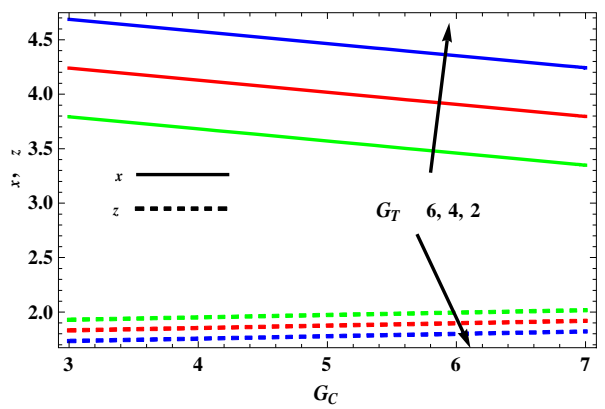

(a) Uniform plate temperature

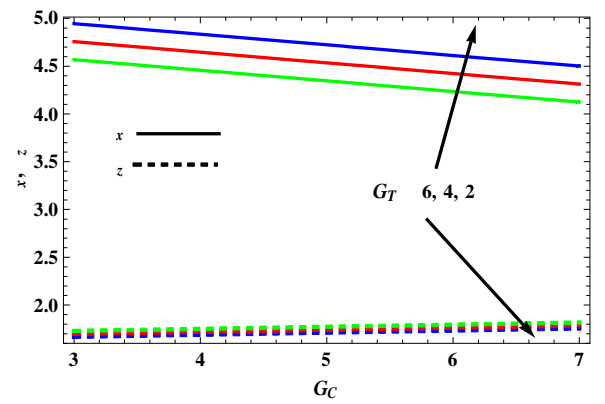

(b) Ramped plate temperature

Fig. 18 Skin friction distribution when $m=0.75, K^{2}=1, M^{2}=9$, $k_{1}=0.3, P_{r}=0.71, S_{c}=0.22, \phi=1, K_{1}=0.2$ and $t=0.5$ : (a) uniform plate temperature and (b) ramped plate temperature.

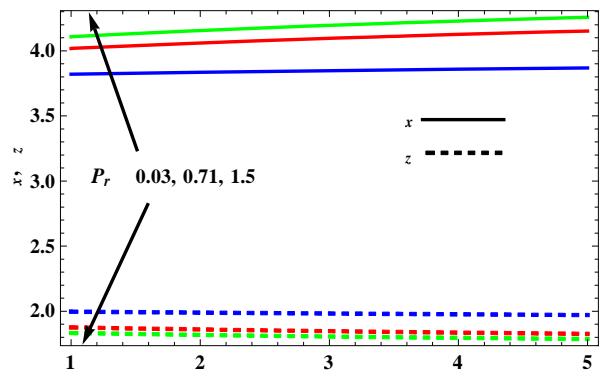

(a) Uniform plate temperature

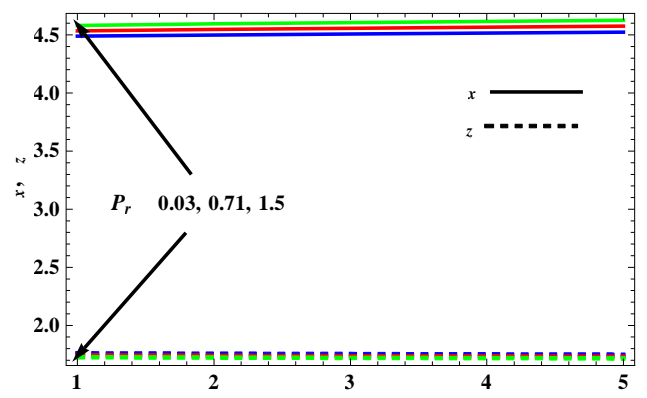

(b) Ramped plate temperature

Fig. 19 Skin friction distribution when $m=0.75, K^{2}=1, M^{2}=9$, $k_{1}=0.3, G_{T}=4, G_{C}=5, S_{c}=0.22, K_{1}=0.2$ and $t=0.5$ : (a) uniform plate temperature and (b) ramped plate temperature.

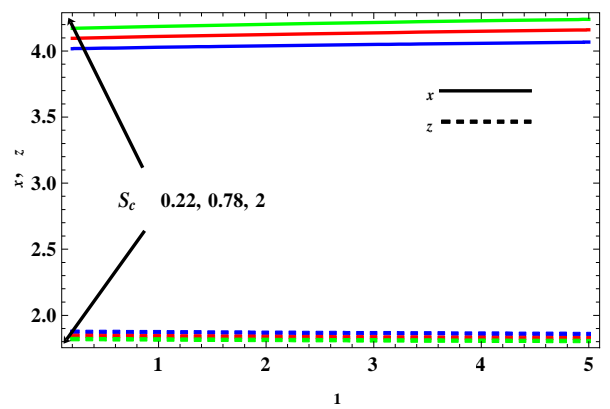

(a) Uniform plate temperature

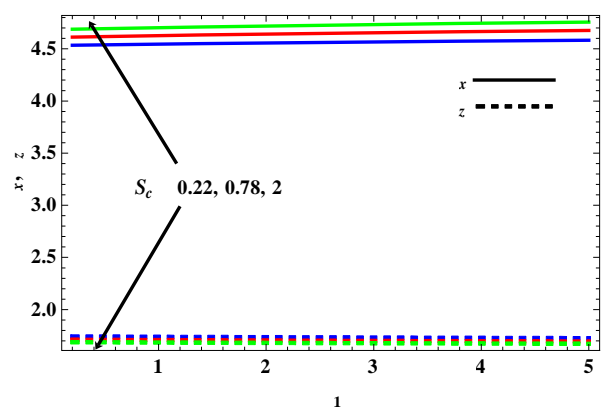

(b) Ramped plate temperature

Fig. 20 Skin friction distribution when $m=0.75, K^{2}=1, M^{2}=9$, $k_{1}=0.3, G_{T}=4, G_{C}=5, P_{r}=0.71, \phi=1$ and $t=0.5$ : (a) uniform plate temperature and (b) ramped plate temperature.

Figs. 19 exhibit that in case of both the uniform and ramped plate temperature, skin friction in the primary flow direction increases whereas skin friction in the secondary flow direction decreases on increasing both the Prandtl number $P_{r}$ and heat absorption parameter $\phi$, which indicates that thermal diffusion tends to reduce skin friction in the primary flow direction whereas heat absorption has reverse effect on it. These effects are reversed on the skin friction in the secondary flow direction.Figs. 20 illustrate that in case of both the uniform and ramped plate temperature, skin friction in the primary flow direction increases whereas skin friction in the secondary flow direction decreases on increasing both the Schemidt number $S_{C}$ and chemical reaction parameter $K_{1}$, which concludes that mass diffusion tends to reduce skin friction in the primary flow direction whereas chemical reaction has reverse effect on it. These effects are reversed on the skin friction in the secondary flow direction.

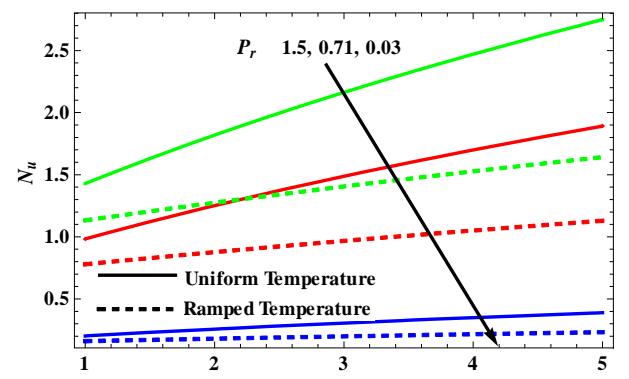

Fig. 21 Nusselt number distribution in case of both uniform and ramped plate temperature. 
Fig. 21 represents that in case of both the uniform and ramped plate temperature, Nusselt number $N_{u}$ increases on increasing both the Prandtl number $P_{r}$ and heat absorption parameter $\phi$. This implies that thermal diffusion tends to reduce rate of heat transfer at the plate whereas heat absorption has reverse effect on it. Fig. 22 shows that in case of both the uniform and ramped plate temperature, Sherwood number $S_{h}$ increases on increasing both the Schemidt number $S_{c}$ and chemical reaction parameter $K_{1}$, this implies that mass diffusion tends to reduce the rate of mass transfer at the plate whereas chemical reaction has reverse effect on it.

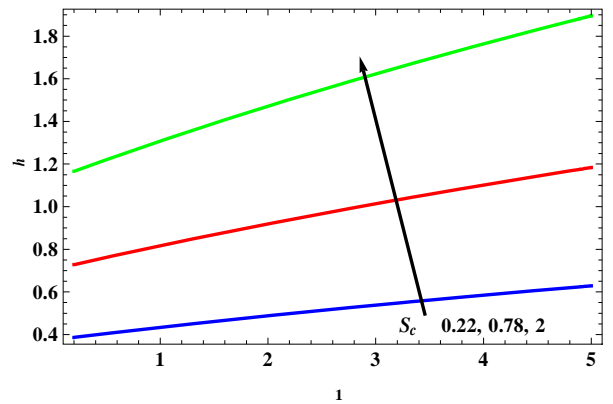

Fig. 22 Sherwood number distribution in case of both uniform and ramped plate temperature.

\section{CONCLUSIONS}

A mathematical analysis has been presented for unsteady hydromagnetic heat and mass transfer natural convection flow past an exponentially accelerated vertical plate in a uniform porous medium taking Hall current and rotation into account with variable species concentration and uniform/variable plate temperature. Hall current tends to enhance fluid velocity in the primary flow direction whereas Coriolis force and magnetic field have reverse effect on it. Both the Hall current and Coriolis force tend to enhance fluid velocity in the secondary flow direction. Magnetic field tends to enhance fluid velocity in the secondary flow direction in the vicinity of the plate whereas this effect is reversed in the region away from the plate. Thermal buoyancy force, species concentration buoyancy force, thermal diffusion and mass diffusion tend to enhance fluid velocity in both the primary and secondary flow directions whereas Darcian drag force, heat absorption and chemical reaction have reverse effect on these. Thermal diffusion tends to enhance fluid temperature whereas heat absorption has reverse effect on it. Mass diffusion tends to enhance species concentration whereas chemical reaction has reverse effect on it. Both the Coriolis force and magnetic field tend to enhance skin friction in both the primary and secondary flow directions. Hall current, thermal buoyancy force, thermal diffusion and mass diffusion tend to reduce skin friction in the primary flow direction whereas Darcian drag force, heat absorption and chemical reaction have reverse effect on it. These effects are reversed on the skin friction in the secondary flow direction. Thermal diffusion tends to reduce the rate of heat transfer at the plate whereas heat absorption has reverse effect on it. Mass diffusion tends to reduce the rate of mass transfer at the plate whereas chemical reaction has reverse effect on it. For small thermal diffusion, the free stream value of velocity and temperature are achieved nearer to the plate in comparison to that for large thermal diffusion. When Darcian drag force is strong, the skin friction in the primary flow direction sharply decreasing whiles the skin friction in the secondary flow direction sharply increasing.

\section{ACKNOWLEDGEMENTS}

Authors are thankful to the reviewers for their valuable comments and suggestions, which helped us to improve the worth of the research work. One of the authors S. Ghousia Begum is thankful to UGC, New Delhi (India) for providing financial assistance to carry out this research work.

\section{NOMENCLATURE}

non-dimensional species concentration

$$
\text { species concentration }\left(\mathrm{mol} / \mathrm{m}^{3}\right)
$$

$$
\text { specific heat at constant pressure ( } J / \mathrm{kg} . \mathrm{K})
$$

chemical molecular diffusivity $\left(\mathrm{m}^{2} / \mathrm{s}\right)$

acceleration due to gravity $\left(\mathrm{m} / \mathrm{s}^{2}\right)$

$$
\text { solutal Grashof number }
$$

$$
\text { thermal Grashof number }
$$

thermal conductivity of the fluid $(W / m . K)$

permeability $\left(\mathrm{m}^{2}\right)$

rotation parameter

permeability parameter

chemical reaction parameter

Hall current parameter

magnetic parameter

Nusselt number

$$
\text { Prandtl number }
$$

\section{Greek Symbols}

$\beta_{C} \quad$ volumetric coefficient of thermal expansion $\left(K^{-1}\right)$

$\beta_{T} \quad$ volumetric coefficient of species concentration expansion

$v \quad$ kinematic viscosity $\left(\mathrm{m}^{2} / \mathrm{s}\right)$

$\phi \quad$ heat absorption parameter

$\rho \quad$ fluid density $\left(\mathrm{kg} / \mathrm{m}^{3}\right)$

$\sigma \quad$ electrical conductivity $(s / m)$

$\tau_{x} \quad$ skin friction component in $x$-direction

$\tau_{z} \quad$ skin friction component in $z$-direction

$\theta$ non-dimensional fluid temperature

\section{Subscripts}




\section{REFERENCES}

Bestman, A. R., and Adjepong, S. K. 1988, "Unsteady Hydromagnetic Free-Convection Flow with Radiative Heat Transfer in a Rotating Fluid,” Astrophysics and Space Science, 143(1), 73-80.

http://dx.doi.org/10.1007/BF00636756

Chamkha, A. J., 2004, "Unsteady MHD Convective Heat and Mass Transfer Past a Semi-Infinite Vertical Permeable Moving Plate with Heat Absorption,” International Journal of Engineering Science, 42(2), 217-230.

http://dx.doi.org/10.1016/s0020-7225(03)00285-4

Cramer, K. R., and Pai, S. I., 1973, "Magnetofluid-Dynamics for Engineers and Applied Physicists,” McGraw-Hill, New York.

Das, K., 2012, "Magnetohydrodynamics Free Convective Flow of a Radiating and Chemically Reacting Fluid Past an Impulsively Moving Plate with Ramped Wall Temperature,” Journal of Applied Mechanics, 79(6), 061017 (11 pages).

http://dx.doi.org/10.1115/1.4006462

Hsieh, J. C., Chen, T. S., and Armaly, B. F., 1993, "Nonsimilarity Solutions for Mixed Convection from Vertical Surfaces in Porous Media: Variable Surface Temperature or Heat Flux," International Journal of Heat and Mass Transfer, 36(6), 1485-1493. http://dx.doi.org/10.1016/S0017-9310(05)80059-6

Israel-Cookey, C., and Tay, G., 2002, "Transient Flow of a Radiating Hydromagnetic Fluid Past an Infinite Vertical Plate," Modelling, Measurement and Control B, 71(4), 1-13.

Israel-Cookey, C., Ogulu, A., and Omubo-Pepple, V. B., 2003, "Influence of Viscous Dissipation and Radiation on Unsteady MHD Free-Convection Flow Past an Infinite Heated Vertical Plate in a Porous Medium with Time Dependent Suction," International Journal of Heat and Mass Transfer, 46(13), 2305-2311.

http://dx.doi.org/10.1016/S0017-9310(02)00544-6

Israel-Cookey, C., and Nwaigwe, C., 2010, "Unsteady MHD Flow of a Radiating Fluid over a Moving Heated Porous Plate with TimeDependent Suction," American Journal of Scientific and Industrial Research, 1(1), 88-95.

http://www.scihub.org/AJSIR

Kamel, M. H., 2001, "Unsteady MHD Convection through Porous Medium with Combined Heat and Mass Transfer with Heat Source/Sink,” Energy Converction and Management, 42(4), 393-405. http://dx.doi.org/10.1016/S0196-8904(00)00067-4

Kim, Y. J., 2000, "Unsteady MHD Convective Heat Transfer Past a Semi-Infinite Vertical Porous Moving Plate with Variable Suction,” International Journal of Engineering Science, 38(8), 833-845. http://dx.doi.org/10.1016/S0020-7225(99)00063-4

Kundu, P. K., Das, K., and Acharya, N., 2014, "Flow Features of a Conducting Fluid Near an Accelerated Vertical Plate in Porous Medium with Ramped Wall Temperature," Journal of Mechanics, 30(3), 277288.

http://dx.doi.org/10.1017/jmech.2014.14

Mbeledogu, I. U., and Ogulu, A., 2007, "Heat and Mass Transfer of an Unsteady MHD Natural Convection Flow of a Rotating Fluid Past a Vertical Porous Flat Plate in the Presence of Radiative Heat Transfer," International Journal of Heat and Mass Transfer, 50(9-10), 1902-1908. http://dx.doi.org/10.1016/j.ijheatmasstransfer.2006.10.016

Nandkeolyar, R., Seth, G. S., Makinde, O. D., Sibanda, P., and Ansari, M. S., 2013, "Unsteady Hydromagnetic Natural Convection Flow of a Dusty Fluid Past an Impulsively Moving Vertical Plate with Ramped Temperature in the Presence of Thermal Radiation,” Journal of Applied Mechanics, 80(6), 061003 (9 pages).

http://dx.doi.org/10.1115/1.4023959

Pal, D., and Talukdar, B., 2011, "Combined Effects of Joule Heating and Chemical Reaction on Unsteady Magnetohydrodynamic Mixed Convection of a Viscous Dissipating Fluid over a Vertical Plate in Porous Media with Thermal Radiation," Mathematical and Computer Modelling, 54(11-12), 3016-3036.

http://dx.doi.org/10.1016/j.mcm.2011.07.030

Philliph, K. J., Raju, M. C., Chamkha, A. J., and Varma, S. V. K., 2014, "MHD Rotating Heat and Mass Transfer Free Convective Flow Past an Exponentially Accelerated Isothermal Plate with Fluctuating Mass Diffusion,” International Journal of Industrial Mathematics, 6(4), 297306.

http://ijim.srbiau.ac.ir/

Raptis, A., and Singh, A. K., 1983, "MHD Free Convection Flow Past an Accelerated Vertical Plate," International Communications in Heat and Mass Transfer, 10(4), 313-321.

http://dx.doi.org/10.1016/0735-1933(83)90016-7

Samiulhaq., Khan, I., Ali, F., and Shafie, S., 2012, "MHD Free Convection Flow in a Porous Medium with Thermal Diffusion and Ramped Wall Temperature," Journal of the Physical Society of Japan, 81(4), 044401 (9 pages). http://dx.doi.org/10.1143/JPSJ.81.044401

Sato, H., 1961, "The Hall Effect in the Viscous Flow of Ionized Gas between Parallel Plates under Transverse Magnetic Field," Journal of Physical Society Japan, 16(7), 1427-1435.

http://dx.doi.org/10.1143/JPSJ.16.1427

Seth, G. S., and Ansari, M. S., 2010, “MHD Natural Convection Flow Past an Impulsively Moving Vertical Plate with Ramped Wall Temperature in the Presence of Thermal Diffusion with Heat Absorption," International Journal of Applied Mechanics and Engineering, 15(1), 199-215.

Seth, G. S., Nandkeolyar, R., and Ansari, M. S., 2013, "Effects of Radiation and Rotation on Unsteady Hydromagnetic Free Convection Flow Past an Impulsively Moving Vertical Plate with Ramped Temperature in a Porous Medium," Journal of Applied Fluid Mechanics, 6(1), 27-38.

Seth, G. S., Hussain, S. M., and Sarkar, S., 2014, "Effects of Hall Current and Rotation on Unsteady MHD Natural Convection Flow with Heat and Mass Transfer Past an Impulsively Moving Vertical Plate in the Presence of Radiation and Chemical Reaction,” Bulgarian Chemical Communications, 46(4), 704-718.

Seth, G. S., Tripathi, R., and Sherma, R., 2015, "Natural Convection Flow Past an Exponentially Accelerated Vertical Ramped Temperature Plate with Hall Effects and Heat Absorption," International Journal of Heat and Technology, 33(3), 139-144. http://dx.doi.org/10.18280/ijht.330321 